\title{
Evaluation of Alveolar Ridge Dimensions Using Various Techniques Prior to Implant Placement: A Comparative Study
}

\author{
Mohammad Jalaluddin ${ }^{1}$, George Sam², Mohamed Abd-Ellatif El-Patal ${ }^{3}$, Narendra V Penumatsa ${ }^{4}$, Abdulfatah Aladmah ${ }^{5}$,
} Prashant Punde ${ }^{6}$

\begin{abstract}
Aim: The present study aimed to assess the dimensions of alveolar ridge using different techniques before placement of implant.

Materials and methods: Totally 20 patients were chosen for this study. The study was divided into three groups based on the method of measurements of width of alveolar ridge, and all the procedures were performed in all the groups. Group I: Using cone-beam computed tomography (CBCT), group II: ridge mapping dimensions before reflection of surgical flap, group III: direct caliper measurements after surgical exposure of the bone. A transparent acrylic resin stent was made on the study model with reference points. The reference points were seen over the stent through the clear acrylic resin.

Results: The mean alveolar ridge dimensions were $4.20 \pm 1.80 \mathrm{~mm}, 3.89 \pm 2.24 \mathrm{~mm}$, and $4.24 \pm 0.94 \mathrm{~mm}$ with the use of CBCT, ridge mapping dimension before surgical flap reflection, and direct caliper measurements after surgical exposure of the bone, respectively, at point 1 ; and $7.68 \pm 1.46 \mathrm{~mm}, 6.10 \pm 1.88 \mathrm{~mm}, 6.90 \pm 1.32 \mathrm{~mm}$, respectively, at point 2 . A statistically significant difference was found between the groups at point 2 by an analysis of covariance. Also, the difference between groups I and II was statistically significant.

Conclusion: Within the limitations of this study, it was concluded that CBCT and ridge mapping measurements when compared individually with the gold standard surgical open method, CBCT proved to be a highly specific and sensitive method detecting the residual alveolar ridge width in the treatment planning of dental implants.

Clinical significance: Implants have become the imminent replacement option for missing teeth. The surgical procedure necessitates a prior knowledge of the surgical site and planning of treatment. Measurement of the residual alveolar bone dimensions is an important precondition for placement of dental implants and their success.
\end{abstract}

Keywords: Alveolar ridge, Cone-beam computed tomography, Dental implant, Ridge mapping.

World Journal of Dentistry (2020): 10.5005/jp-journals-10015-1749

\section{INTRODUCTION}

Dental implants are routinely used as a conventional replacement therapy for missing teeth, ranging from single missing tooth to partial and/or complete edentulism. The use of dental implants as a supportive prosthodontic restoration has shown successful results. To achieve long-term success of dental implants, evaluation of the dimensions of the resorbing alveolar process must be accurate because an implant should be surrounded by at least $1 \mathrm{~mm}$ of bone. A favorable outcome can be achieved by detailed diagnosis and treatment planning. ${ }^{1}$

The most imperative factor for diagnosis before the placement of implant is to measure the height and width of the residual alveolar bone. Any error in preoperative measurement of the residual alveolar bone may result in a crucial state after reflecting the flap. It may lead to modification in the treatment plan at the time of implant surgery or may also result in implants not being placed. Such an awkward condition can be avoided by adapting various appropriate clinical and radiographic procedures to measure the alveolar bone extent prior to implant placement. $^{2}$

Several clinical and radiographic studies have demonstrated a reduction in width and height of the alveolar bone after tooth extraction resulting in inadequate horizontal and vertical dimensions of the alveolar ridge. The reduced alveolar ridge height and width impacts placement of implant, its stability, and success. After surgically exposing the bone, the width of buccolingual part of alveolar ridge can be assessed using computed tomography,
${ }^{1}$ Department of Periodontics and Oral Implantology, Kalinga Institute of Dental Sciences, KIIT (Deemed to be University), Bhubaneswar, Odisha, India

2,4,5 Department of Preventive Dental Sciences, College of Dentistry, Prince Sattam Bin Abdul Aziz University, Alkharj, Kingdom of Saudi Arabia

${ }^{3}$ Department of Pedodontics and Oral Health, College of Dentistry, Al Azhar University, Cairo, Egypt

${ }^{6}$ Department of Oral and Maxillofacial Surgery, School of Dental Sciences, Krishna Institute of Medical Sciences (Deemed to be University), Karad, Maharashtra, India

Corresponding Author: Mohammad Jalaluddin, Department of Periodontics and Oral Implantology, Kalinga Institute of Dental Sciences, KIIT (Deemed to be University), Bhubaneswar, Odisha, India, Phone: +91 9338131843, e-mail: drjalal1979@gmail.com

How to cite this article: Jalaluddin M, Sam G, Abd-Ellatif El-Patal M, et al. Evaluation of Alveolar Ridge Dimensions Using Various Techniques Prior to Implant Placement: A Comparative Study. World J Dent 2020;11(4):299-303.

Source of support: Nil

Conflict of interest: None

trans-tomography, ridge mapping, ultrasonography, and direct caliper measurement. $^{3}$

Safety measures have to be employed for different parts of the jaws during implantation procedure. Tooth loss results in a 
specific pattern of alveolar ridge reduction such as, alteration in the angle of the residual ridge, and thinning of crestal bone resulting in weakening of the alveolar ridge. ${ }^{4}$ The modified residual ridge anatomy results in problems during surgery, including inadequate thickness of labial bone or precise positioning of implant. ${ }^{5}$ Thus, the present study was aimed to compare and assess the dimension of alveolar ridge using cone-beam computed tomography (CBCT), ridge mapping dimension before reflection of surgical flap, and direct caliper measurements after exposing the bone surgically before the placement of implant.

\section{Materials and Methods}

\section{Study Sample}

The present study was conducted in the Department of Periodontics, Kalinga Institute of Dental Sciences, Bhubaneswar, India. Total 23 cases were selected for the study, and 3 cases were excluded during treatment planning procedure. Finally, 20 patients were chosen for this trial. Patients signed a consent form after being informed about the criteria of the proposed study, including potential risks and benefits and alternative treatment option.

The presence of one or more periodontally stable and healthy posterior tooth next to the edentulous ridge as a support for radiographic stent in partially edentulous patients, at least 3 months of healing period post tooth extraction and presence of good oral hygiene were the inclusion criteria. Pregnant women, participant with debilitating diseases, immunocompromised condition, and smoking were excluded from the study.

The study was divided into three groups based on the method of measurements of width of alveolar ridge.

Group I: Using CBCT

Group II: Ridge mapping dimension before reflection of surgical flap

Group III: Direct caliper measurements after exposing the bone surgically

\section{Fabrication of Stent}

Preliminary mandibular and maxillary diagnostic impressions were made with alginate and models were poured with dental stone. First mark was made on the ridge crest (reference point) in reference to the next teeth. Later one point (point 1) was marked horizontally at a distance of $3 \mathrm{~mm}$ from the reference point and another point (point 2) was marked at a distance of $6 \mathrm{~mm}$ distance from the reference point. Points 1 and 2 were marked on both buccal and palatal/lingual aspect, respectively. A clear acrylic resin stent was made over the study model having reference points. A micro-motor with a straight fissure bur was used to drill holes of $1 \mathrm{~mm}$ diameter on the buccal, lingual/palatal, and crestal side of the stent over the reference points that were visible through the transparent acrylic resin material. The holes were later filled with gutta-percha points till the end of the tissue surface. After the patients wore the stent, a computed tomography and a cone-beam computed tomography were taken. The gutta-percha was seen as a radio-opaque material in the tomography and served as the radiological reference site for measurement.

\section{Group I: Using Cone-beam Computed Tomography (CBCT)}

A 5\% solution of povidone iodine was used to disinfect acrylic stents. Patients wore acrylic stent and were later exposed. Patient was in supine position and exposed to CBCT with a $130 \mathrm{kV} 64 \mathrm{~mA}$ exposure rate for 22.7 seconds with a thickness of $0.63 \mathrm{~mm}$ slice (Fig. 1). For ridge measurement, an appropriate site where the guttapercha was most easily seen on the CBCT was chosen.

\section{Group II: Ridge Mapping Dimensions Prior to Surgical Flap Reflection}

Local anesthesia was administered to the patients. After this, the disinfected acrylic stent was positioned on the alveolar ridge which is edentulous. Ridge mapping was done using a calibrated periodontal probe. The probe was entered through mucosa of the oral cavity till the bone was touched (Fig. 2). After this, probe was used to remove the stent and located on the sectioned model. A marking was made on the cast for the depth of insertion of the probe and the dotted line was linked. This line provided alveolar bone's surface topography.

\section{Group III: Direct Caliper Measurements Following Surgical Exposure of the Bone}

Once these two techniques were performed, surgical blade, and periosteal elevator were used to reflect a surgical flap during the placement of implant and width of residual alveolar ridge was measured for all the patients, directly on the uncovered bone at different locations of the ready guiding holes using same surgical stent with the help of bone caliper (Fig. 3), and these measurements were noted by single principal investigator and further analyzed. And all the procedures were performed in all the three groups.

\section{Statistical Analysis}

A windows version SPSS 20.0 was used to perform the statistical procedure. The values obtained were entered in a table, and one-way analysis of variance (ANOVA) test was used to statistically analyze individual comparisons, and Tukey's HSD test was used for multiple comparisons among the groups. A $p$ value less than 0.05 is considered statistically significant.

\section{RESULTS}

The mean and standard deviation of dimensions of alveolar ridge got from all the three techniques is as shown in Table 1, and they are $4.20 \pm 1.80 \mathrm{~mm}, 3.89 \pm 2.24 \mathrm{~mm}$, and $4.24 \pm 0.94 \mathrm{~mm}$ with the use of $C B C T$, ridge mapping dimension before reflection of surgical flap, and direct caliper measurements after surgical exposure of the

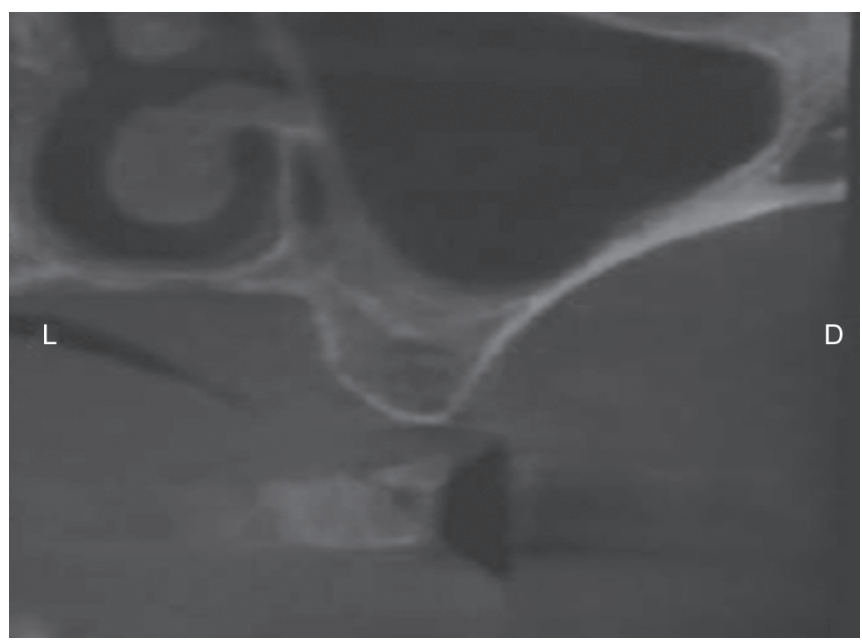

Fig. 1: Alveolar ridge width measurements in cone-beam computed tomography at 26 region 


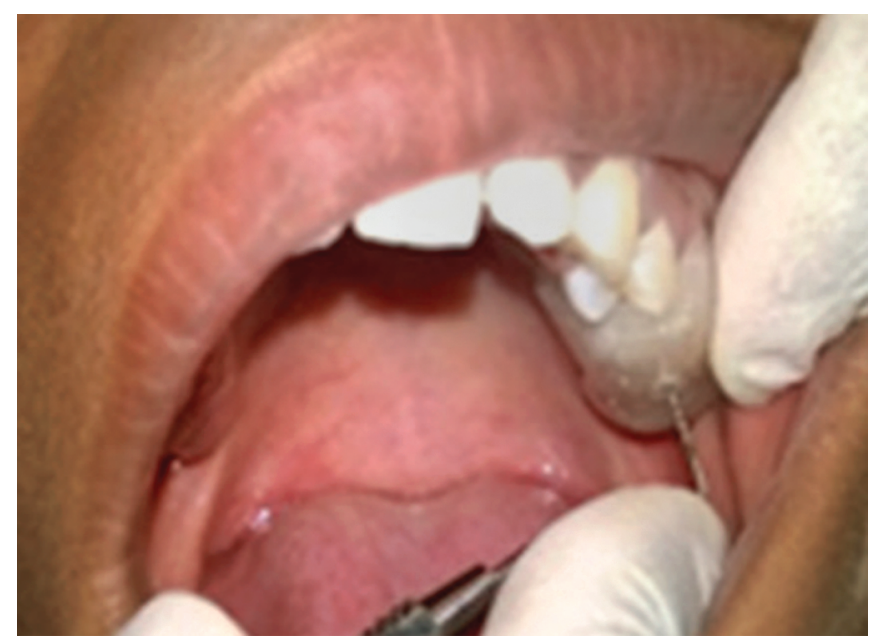

Fig. 2: Ridge mapping dimension prior to surgical flap reflection at 26 region

Table 1: Mean and standard deviation of alveolar ridge dimensions obtained from all the three techniques

\begin{tabular}{|c|c|c|}
\hline Groups & Reference points & $\operatorname{Mean}(\mathrm{mm}) \pm S D$ \\
\hline \multirow{2}{*}{$\begin{array}{l}\text { Group I: Using cone-beam } \\
\text { computed tomography } \\
\text { (CBCT) }\end{array}$} & Point 1 & $4.20 \pm 1.80$ \\
\hline & Point 2 & $7.68 \pm 1.46$ \\
\hline \multirow{2}{*}{$\begin{array}{l}\text { Group II: Ridge mapping } \\
\text { dimensions prior to surgical } \\
\text { flap reflection }\end{array}$} & Point 1 & $3.89 \pm 2.24$ \\
\hline & Point 2 & $6.10 \pm 1.88$ \\
\hline \multirow{2}{*}{$\begin{array}{l}\text { Group III: Direct caliper meas- } \\
\text { urements following surgical } \\
\text { exposure of the bone }\end{array}$} & Point 1 & $4.24 \pm 0.94$ \\
\hline & Point 2 & $6.90 \pm 1.32$ \\
\hline
\end{tabular}

Table 2: Mean comparison of alveolar ridge dimensions obtained from all the three techniques at point 1

\begin{tabular}{lclll}
\hline Groups & Mean $\pm S D$ & Std. error & $F$ & p value \\
\hline $\begin{array}{l}\text { Group I: Using cone- } \\
\text { beam computed }\end{array}$ & $4.20 \pm 1.80$ & 0.1044 & 22.310 & 0.831 \\
tomography (CBCT) & & & & \\
$\begin{array}{l}\text { Group II: Ridge map- } \\
\text { ping dimensions }\end{array}$ & $3.89 \pm 2.24$ & 0.0229 & & \\
prior to surgical flap \\
reflection
\end{tabular}

bone, respectively, at point 1 and $7.68 \pm 1.46 \mathrm{~mm}, 6.10 \pm 1.88 \mathrm{~mm}$, $6.90 \pm 1.32 \mathrm{~mm}$, respectively, at point 2 .

The mean comparison of alveolar ridge dimensions from all the three techniques at points 1 and 2 is as shown in Tables 2 and 3. An ANOVA at point 2 showed statistical significance. The mean measurements were almost same between surgical exposure and $\mathrm{CBCT}$, which shows that $\mathrm{CBCT}$ is better than ridge mapping.

Tukey's post hoc test for multiple comparisons of alveolar ridge dimensions at point 2 is as shown in Table 4. Groups I and II demonstrated statistically significant difference.

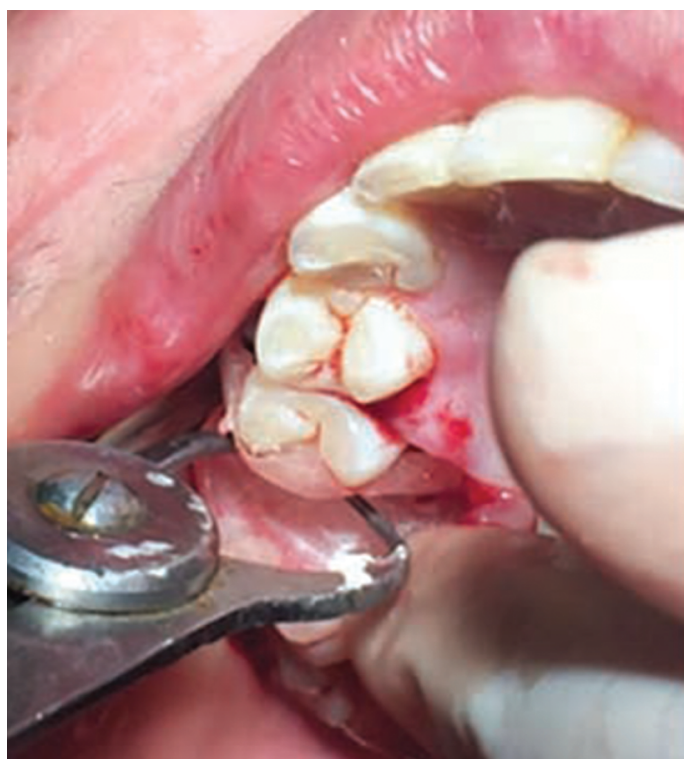

Fig. 3: Direct caliper measurements following surgical exposure of the bone at 16 region

Table 3: Mean comparison of alveolar ridge dimensions obtained from all the three techniques at point 2

\begin{tabular}{|c|c|c|c|c|}
\hline Groups & Mean $\pm S D$ & Std. error & $F$ & $p$ value \\
\hline $\begin{array}{l}\text { Group I: Using cone- } \\
\text { beam computed } \\
\text { tomography (CBCT) }\end{array}$ & $7.68 \pm 1.46$ & 0.0312 & 24.122 & 0.001 \\
\hline $\begin{array}{l}\text { Group II: Ridge map- } \\
\text { ping dimensions } \\
\text { prior to surgical flap } \\
\text { reflection }\end{array}$ & $6.10 \pm 1.88$ & 0.0120 & & \\
\hline $\begin{array}{l}\text { Group III: Direct caliper } \\
\text { measurements follow- } \\
\text { ing surgical exposure } \\
\text { of the bone }\end{array}$ & $6.90 \pm 1.32$ & 0.0022 & & \\
\hline
\end{tabular}

Table 4: Multiple comparisons using Tukey's post hoc test

\begin{tabular}{llcl}
\hline Groups & Compared with & Mean difference & Sig. \\
\hline Group I & Group II & 1.58 & 0.001 \\
& Group III & 0.78 & 0.326 \\
Group II & Group I & -1.58 & 0.001 \\
& Group III & -0.80 & 0.120 \\
Group III & Group I & -0.78 & 0.328 \\
& Group II & 0.80 & 0.122 \\
\hline
\end{tabular}

The inference of the present study indicates that the ridge mapping and CBCT measurements when exclusively compared with the surgical open method, the gold standard. CBCT proved to be a highly accurate method detecting the residual alveolar ridge width than ridge mapping method in the dental implant treatment planning.

\section{Discussion}

The best choice for replacement of missing teeth is dental implants. The success of dental implants depends on the quality and quantity 
of residual jaw bone; thus, precise measurement of alveolar process is important. In the surgical design of preimplants, the morphology and width and height of alveolar bone are assessed to evaluate the quality of existing bone. After recording the dimensions of residual alveolar bone, the important fixture that can appropriately offer highest distribution of masticatory forces and support is chosen. Many morphological defects such as undercuts in bone that are not easily identified during clinical examinations are seen with crosssectional imaging. In mandible, mylohyoid ridge is an important anatomic landmark. An undercut is seen under the mylohyoid ridge. The ridge nearly fades in the anterior region and is sharp and evident in molar areas. ${ }^{6}$

Radiographic assessment of the jaws before operative procedure is considered important in planning treatment for implant-supported prostheses. Although panoramic radiograph provides a complete view of the jaws, it is not complete due to the varying magnification and distortions that it creates. Intraoral periapical radiographs of the edentulous jaw display the height of bone, the mesiodistal space between root, as well as the location of the anatomical structures in a bucco-lingual plane. However, these diagnostic methods do not provide any details related to perfect orientation and sagittal bone morphology to provide the implant needed to meet restorative requirements. The quality and quantity of residual alveolar bone will impact the selection of implants with regard to their length, number, type, and diameter. Placement of implants mostly needs a more wide-ranging radiographic examination than that used for other types of oral treatment procedures. ${ }^{7}$

Therefore, $C B C T$ is essential for three-dimensional view of the bone morphology of that particular region where we want to position the implant. The benefits of CBCT-based systems are superior contrast of image with a completely distinct image layer with no blurring and even enlargement. The drawbacks of CBCT include high-cost, restricted accessibility to reconstructive software, increased radiation dose in comparison with conventional tomography. ${ }^{8}$

The results obtained in the present study did not show any significant difference between direct surgical exposure and $C B C T$ dimensions of ridges. This shows in the present study CBCT proved to be a highly accurate method detecting the residual alveolar ridge width than ridge mapping method in the dental implant treatment planning. This contradicts with the results obtained by Chen et al. ${ }^{9}$ who found consistent buccolingual measurements with ridge mapping and direct caliper and concluded that CBCT did not offer any further substantial diagnostic data related to site of implant placement. Studies by Castro-Ruiz et al., ${ }^{10}$ Chugh et al., ${ }^{11}$ and Luk et al. ${ }^{12}$ found ridge mapping to be a precise indicator of width of residual bone when compared to different radiographic methods. Wahed et al. ${ }^{13}$ detailed that the precision of diagnostic information obtained with a CBCT relies on the method of use of the system and modification of the obtained images.

The width of the ridge can also be measured by ridge mapping calipers method. This method involves entering the buccal and lingual mucosa until bone (after local anesthesia administration) with calipers fabricated for this purpose. A range of measurements of the planned implant placement site are made before mucoperiosteal flap reflection. This method has been promoted by Wilson ${ }^{14}$ and Traxler et al., ${ }^{15}$ who propose that it is a consistent and suitable method for evaluating correctness of probable implant sites. The advantages of ridge mapping method are lack of radiation exposure to patient and its ease of use.

As per the findings of this study, no significant difference exists between ridge mapping measurements and direct surgical exposure. Also, this study promotes ridge mapping method for the assessment of width of alveolar ridge for partially edentulous ridges which is in accordance with the study done by Veyre-Goulet et al. ${ }^{16}$

However, future studies enrolling larger sized samples are needed to confirm the results obtained by this study, as the present study included a population based on convenient sampling method, a single observer assessed the data, and the precision of materials used in this study could affect the data.

\section{CONCLUSION}

After considering the drawbacks of this study, it was determined that ridge mapping and $\mathrm{CBCT}$ measurements when exclusively compared with the surgical open method, the gold standard, CBCT was demonstrated to be extremely sensitive and specific method in measuring the width of residual alveolar ridge in planning the treatment with dental implants.

\section{References}

1. Chowdhary R, Chandraker N. Simple diagnostic method to assess the available bone for immediate implant placement in an extracted socket. J Oral Implantol 2011;37(4):473-476. DOI: 10.1563/AAIDJOI-D-09-00111.

2. Williams MY, Mealey BL, Hallmon WW. The role of computerized tomography in dental implantology. Int J Oral Maxillofac Implants 1992;7(3):373-380.

3. Allen F, Smith DG. An assessment of the accuracy of ridge mapping in planning implant therapy for the anterior maxilla. Clin Oral Implants Res 2000;11(1):34-38. DOI: 10.1034/j.1600-0501.2000.011001034.x.

4. Tyndall DA, Rathore S. Cone-beam CT diagnostic applications: caries, periodontal bone assessment, and endodontic applications. Dent Clin North Am 2008;52(4):825-841. DOI: 10.1016/j.cden.2008.05.002.

5. Safi Y, Vasegh Z, Kadkhodazadeh M, et al. Different methods to evaluate mandibular alveolar ridge in cone beam computed tomography images in pre-implant surgery assessments. Int J Med Res Health Sci 2016;5(11):134-142.

6. Sutaria FB, Shah DN, Chauhan CJ, et al. Comparative evaluation of various methods of assessing residual alveolar ridge width prior to dental implant placement: an in vivo study. J Dent Implant 2019;9(1):12-19. DOI: 10.4103/jdi.jdi_19_17.

7. Dave $\mathrm{BH}$, Sutaria $\mathrm{S}$, Mehta $\mathrm{S}$, et al. A comparative study of three different methods for evaluating width of alveolar ridge prior to implant placement: an in vivo study. Int J Oral Care Res 2017;5(1): 53-60. DOI: 10.5005/jp-journals-10051-0082.

8. Kassebaum DK, Nummikoski PV, Triplett RG, et al. Cross-sectional radiography for implant site assessment. Oral Surg Oral Med Oral Pathol 1990;70(5):674-678. DOI: 10.1016/0030-4220(90)90421-n.

9. Chen LC, Lundgren $\mathrm{T}$, Hallstrom $\mathrm{H}$, et al. Comparison of different methods of assessing alveolar ridge dimensions prior to dental implant placement. J Periodontol 2008;79(3):401-405. DOI: 10.1902/ jop.2008.070021.

10. Castro-Ruiz CT, Noriega J, Guerrero ME. Validity of ridge mapping and computed tomography in dental implant therapy. J Indian Soc Periodontol 2015;19(3):290-293. DOI: 10.4103/0972-124X. 154189.

11. Chugh A, Bhisnoi P, Kalra D, et al. Comparative evaluation of three different methods for evaluating alveolar ridge dimension prior to implant placement: an in vivo study. J Dent Implant 2013;3(2):101-110. DOI: 10.4103/0974-6781.118872. 
12. Luk LC, Pow EH, Li TK, et al. Comparison of ridge mapping and cone beam computed tomography for planning dental implant therapy. Int J Oral Maxillofac Implants 2011;26(1):70-74.

13. Abdel-Wahed NA, Hamdy RM, Abdel-Latif ZA. Measurements of jaw bones for implant site assessment using cone-beam computed tomography: interobserver and intraobserver agreement. Egypt J Oral Maxillofac Surg 2012;3(2):62-69. DOI: 10.1097/01. OMX.0000418694.73046.c1.

14. Wilson DJ. Ridge mapping for determination of alveolar ridge width. Int J Oral Maxillofac Implants 1989;4(1):41-43.
15. Traxler $M$, Ulm C, Solar $P$, et al. Sonographic measurement versus mapping for determination of residual ridge width. J Prosthet Dent 1992;67(3):358-361. DOI: 10.1016/0022-3913(92) 90246-7.

16. Veyre-Goulet S, Fortin T, Thierry A. Accuracy of linear measurement provided by cone beam computed tomography to assess bone quantity in the posterior maxilla: A human cadaver study. Clin Implant Dent Relat Res 2008;10(4):226-230. DOI: 10.1111/j.17088208.2008.00083.x. 